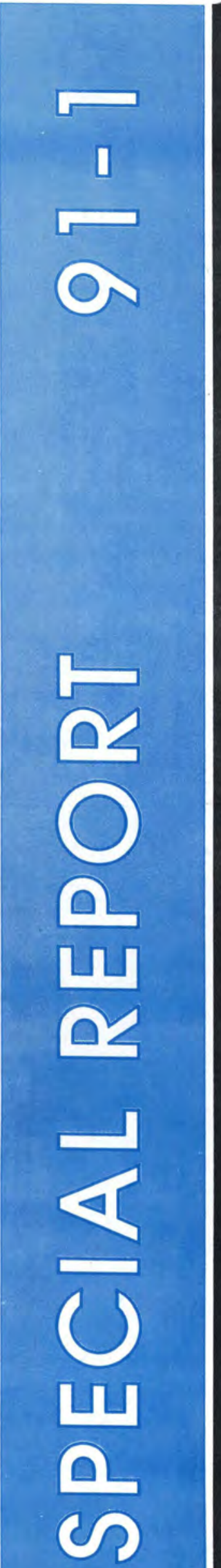

A Membrane For In-Situ Optical Detection of Organic Nitro Compounds Based On Fluorescence Quenching

W. Rudolf Seitz, Chen Jian and Donald C. Sundberg January 1991 
For conversion of SI metric units to U.S./British customary units of measurement consult ASTM Standard E380, Metric Practice Guide, published by the American Society for Testing and Materials, 1916 Race St., Philadelphia, Pa. 19103.

This report is printed on paper that contains a minimum of $50 \%$ recycled material. 


\section{Special Report 91-1}

U.S. Army Corps of Engineers

Cold Regions Research \& Engineering Laboratory
A Membrane For In-Situ
Optical Detection of
Organic Nitro Compounds
Based On Fluorescence Quenching

W. Rudolf Seltz, Chen Jian and Donald C. Sundberg 


\section{PREFACE}

This report was prepared by W. Rudolf Seitz, Chen Jian and Donald C. Sundberg, Departments of Chemistry and Chemical Engineering, University of New Hampshire (UNH). This research was supported financially by the U.S. Army Toxic and Hazardous Materials Agency (USATHAMA) through the U.S. Army Cold Regions Research and Engineering Laboratory (contract DACA89-89-K-0004). The authors thank Dr. Thomas Jenkins for helpful discussions.

Technical review was provided by Dr, Thomas Jenkins, CRREL, and Dr. C.L. Grant, UNH.

The contents of this report are not to be used for advertising or promotional purposes. Citation of brand names does not constitute an official endorsement or approval of the use of such commercial products. 


\title{
A Membrane For In-Situ Optical Detection of Organic Nitro Compounds Based On Fluorescence Quenching
}

\author{
W. RUDOLF SEITZ, CHEN JIAN AND DONALD C. SUNDBERG
}

\section{INTRODUCTION}

In the past, unsound explosive disposal practices have led to contamination of yarious sites by nitrated organics. Because these compounds partition significantly into water from soil, they can migrate in groundwater, posing a potential health hazard. Groundwater monitoring for several different nitrated organics is required to evaluate the extent of this problem. Hexahydro-1,3,5-trinitro-1,3,5-triazine (RDX) and 2,4,6-trinitrotoluene (TNT) are the two most important because they are manufacture and used in larger quantities than other explosives. RDX is of greater concern because it is stable and migrates more rapidly in groundwater,

Current practice is to get samples from monitoring wells and to bring them back to the laboratory for analysis. This is expensive and subject to potential error owing to contamination or instability of samples. As a consequence, there is a need for rapid, inexpensive screening methods that function in situ. Even if these methods do not yield definitive analytical results, they will be useful if they identify those samples that need to be brought back to the laboratory for comprehensive analysis. Our goal has been to develop solid phase indicators that can be used for in-situ determination of nitrated organics. We have previously described a membrane that reacts with polynitroaromatic hydrocarbons to form a brown product, which can be remotely detected through fiber optics (Zhang et al. 1988a and b, Zhang et al. 1989a and b, Zhang and Seitz 1989). Because the reaction to form the brown product is irreversible, it is possible to achieve detection limits in the low parts-per-billion range by waiting for product to accumulate to a detectable level. However, this membrane can not be used for continuous in-situ measurements, and it does not respond to nitramines, another important class of explosives.
We report here a new approach to in-situ optical detection of explosives based on quenching of fluorescence by nitro compounds. A fluorophor is incorporated into a plasticized polymeric membrane, which preconcentrates organic nitro compounds from water. This leads to fluorescence quenching. This process is reversible and responds to all organic nitro compounds, including nitramines, which are extracted by the membrane. However, it is only sensitive to concentrations in the low parts-per-million range and does not provide a means of distinguishing between differentorganic nitro compounds. It will be most useful for screening samples for the presence of explosives. The preparation and response characteristics of fluorescent membranes that respond to nitro compounds are described in this report. Fluorescence from the membranes was measured remotely through fiber optics. However, we were not successful in measuring fluorescence lifetimes through fiber optics because the time constant of the detection system was too long.

\section{EXPERIMENTAL}

\section{Reagents}

All polymers and plasticizers were obtained from Scientific Polymer Products, Inc., and all fluorophors were obtained from Molecular Probes, Inc. Water was purified by deionization followed by distillation. It did not produce a detectable blank. Organic solvents were ACS reagent grade; 4-cyanophenyl 4-heptylbenzoate was obtained from Aldrich, while RDX, TNT and 2,4dinitrotoluene (DNT) were provided by the U.S. Army Toxic and Hazardous Materials Agency.

\section{Instrumentation}

Fluorescence was measured with an SLM 8000 spectrofluorometer system. Absorbance was measured 


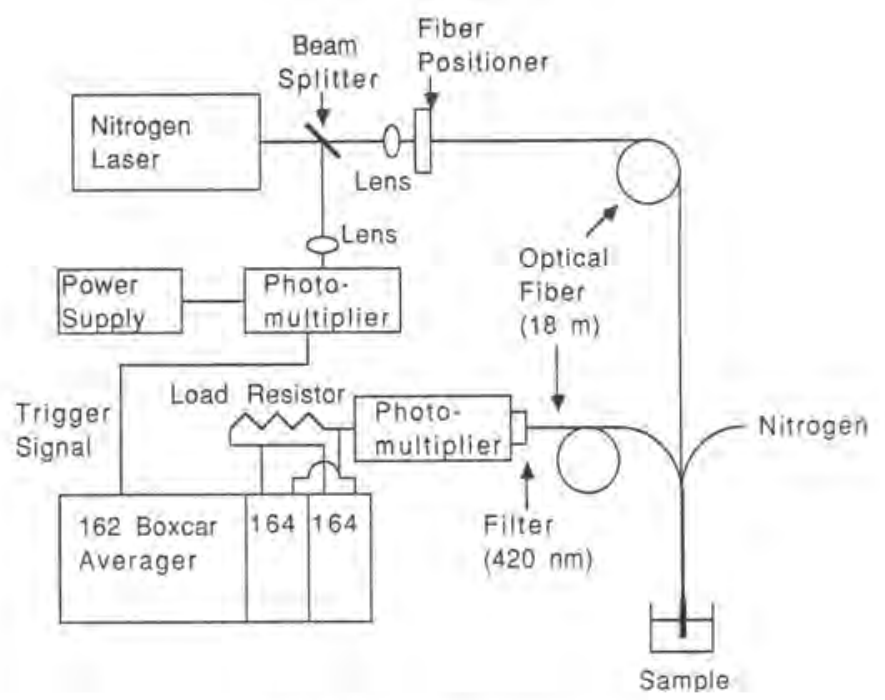

Figure 1. Laser spectrometer.

with a Spectronic 200 double beam spectrophotometer from Shimadzu. Figure 1 shows a block diagram of a system that we developed to measure fluorescence lifetimes in situ through fiber optics. The excitation source is a Phase-R MICRO nitrogen laser that emits pulsed radiation at $337.1 \mathrm{~nm}$. Peak power is $150 \mathrm{~kW}$ and and the pulse width is $1 \mathrm{~ns}$, full width half maximum. The signal from the nitrogen laser is focused onto a fused silica optical fiber (Superguide $G$ from Fiberguide Industries, Inc.) having a 0.40 -mm core diameter. Light from the nitrogen laser was transmitted through $18 \mathrm{~m}$ of fiber to the membrane in the sample. A second 18-m length of fiber was used to transmit fluorescence from the membrane to a P-8850 photomultiplier tube that serves as the detector. The current from the photomultiplier passes through a load resistor that converts the signal to a voltage. This signal is divided so that it enters both model 164 gated integrators in a model 162 boxcar averager (all from Princeton Applied Research).

A piece of quartz serves as a beam splitter, diverting approximately $4 \%$ of the laser light onto a IP28 photomultiplier tube. The signal from this detector triggers the boxcar, opening the gated integrators. The transit time through the optical fibers is approximately $120 \mathrm{~ns}$. Because this is longer than the 75-ns dead time between the arrival of the trigger pulse and the arrival of the signal at the gated integrators, the gate is open by the time the signal arrives. The following settings were used on the gated integrators: aperture delay range$1,000 \mathrm{~ns}$, aperture duration- $-50 \mathrm{~ns}$, and time constant$1 \mu \mathrm{s}$. The two gated integrators were set to open at different times after arrival of the fluorescent signal.
Figure 2 shows a close-up of the arrangement at the distal end of the fiber optics. The membrane is held approximately $1 \mathrm{~mm}$ from the ends of the two fibers in a covered cuvette. Nitrogen is bubbled through the cuvette to remove oxygen that otherwise interferes.

\section{Procedures}

We screened fluorophors for susceptibility to RDX quenching by combining $1.0 \mathrm{~mL}$ of 0.0010 -M fluorophor in ethanol with $0,1.0,2.0,4.0$ and $8.0 \mathrm{~mL}$ of $0.0010-\mathrm{M}$ RDX in ethanol $(220 \mathrm{mg} / \mathrm{L})$ and diluting with ethanol to a total volume of $10.0 \mathrm{~mL}$. The fluorescence of these solutions was then compared.

Membranes were prepared by solvent casting. Polymer (typically 0.2 to $0.3 \mathrm{~g}$ ), plasticizer (typically 0.1 to $0.2 \mathrm{~mL}$ ) and fluorophor were dissolved in 6 to $8 \mathrm{~mL}$ of

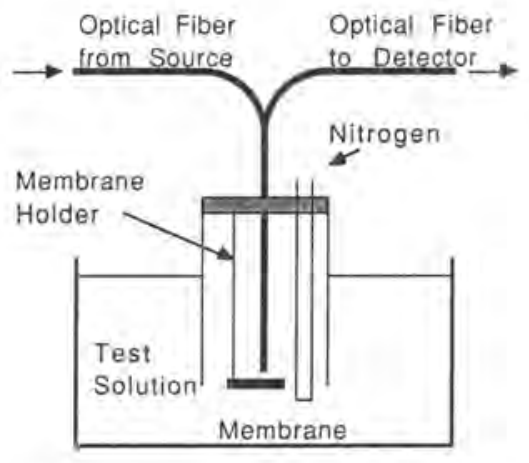

Figure 2. Sample cell arrangement- 
cyclohexanone or tetrahydrofuran. After using a vortex mixer to ensure that the solution was homogeneous, we poured the solution into a glass petri dish $(8-\mathrm{cm}$ diameter) and allowed the solvent to evaporate. This required 24 to 72 hours. The resulting membrane was cut into small sections, each one weighing between 2 to $5 \mathrm{mg}$ and having an area of approximately 0.5 to $0.6 \mathrm{~cm}^{2}$. Membrane thickness was approximately $0.08 \mathrm{~mm}$.

Fluorescence from membranes was measured by holding the membrane between two flat plastic frames that were placed in a $1.00-\mathrm{cm}$ quartz cuvette. The arrangement is shown in Figure 3. A hole in one of the plastic pieces allowed solution in the cuvette to contact the membrane. Fluorescence was measured as a function of time after the membrane contacted solutions of RDX, TNT or DNT. Data are reported as the initial intensity before any quencher has entered the membrane $I_{0}$ divided by the intensity in the presence of quencher $I$. This parameter increases linearly with quencher concentration if Stern-Vollmer quenching conditions apply (Parker 1968).

We measured coefficients for RDX partitioning into membranes by having a known weight of membrane contact a known weight of aqueous solution containing $5 \mathrm{mg} / \mathrm{L}$ of RDX. After allowing 1 hour for equilibration,

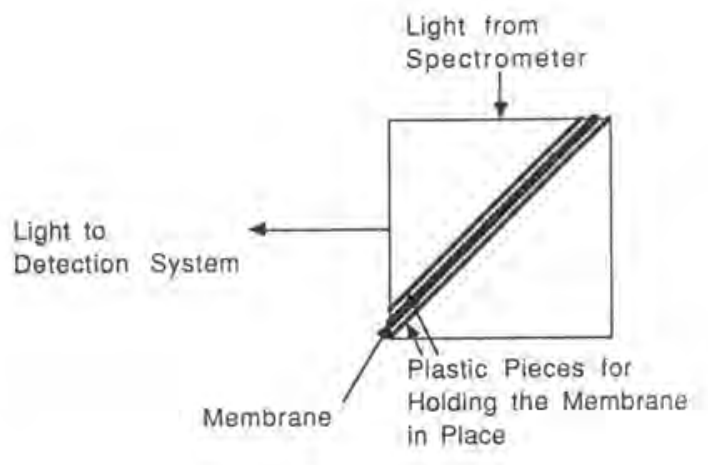

a. Top view.

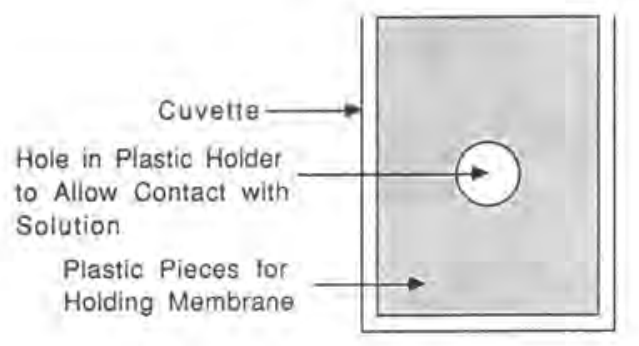

b. Side view.

Figure 3. Arrangement for measuring fluorescence from membranes using spectrofluorometer. we determined the concentration of RDX remaining in the aqueous phase by measuring the absorbance of the aqueous phase at $240 \mathrm{~nm}$. A blank experiment was also conducted in which uv absorbance at $240 \mathrm{~nm}$ was measured after exposing a separate piece of membrane to water for 1 hour. The partition coefficient $K_{\mathrm{p}}$ was calculated from the follawing

$$
K_{\mathrm{p}}=\left[\left(0.35+A_{\mathrm{b}}-A_{\mathrm{s}}\right) / W_{\text {mem }}\right] /\left[\left(A_{\mathrm{s}}-A_{\mathrm{b}}\right) / W_{\text {sol }}\right]
$$

$$
\text { where } \begin{aligned}
A_{\mathrm{s}}= & \text { absorbance of the aqueous RDX after } \\
& \text { equilibration } \\
A_{\mathrm{b}}= & \text { blank absorbance } \\
W_{\text {mem }}= & \text { weight of the membrane } \\
W_{\text {sol }}= & \text { weight of the solution } \\
0.35= & \text { absorbance of } 5.0 \mathrm{mg} / \mathrm{L} \mathrm{RDX} \text { at } 240 \mathrm{~nm} .
\end{aligned}
$$

Note that the partition coefficient calculated by this procedure involves concentrations based on the weights of the two phases rather than volumes.

The procedure to measure the partitioning of RDX from water into plasticizer was the same, except that a known volume of immiscible plasticizer was added to a known volume of aqueous solution. In this case the calculated partition coefficient is based on volumes of the two phases.

The rate at which pyrenebutyric acid (PBA) leaches out of the membrane into aqueous solution was evaluated using a membrane prepared from $0.25 \mathrm{~g}$ of cellulose triacetate, $0.25 \mathrm{~mL}$ of isodecyldiphenylphosphate and $144 \mu \mathrm{g}$ of PBA. Ten sections of a membrane with a combined weight of $55 \mathrm{mg}$ were placed in a capped quartz cuvette containing $2.0 \mathrm{~mL}$ of water and were intermittently shaken. The amount of PBA extracted was determined by comparing fluorescence intensity from the aqueous phase to intensities from aqueous PBA standard solutions.

All measurements were made in air-saturated media, except for an experiment to evaluate the effect of dissolved oxygen on membrane response and the experiments in which fluorescence was measured remotely through fiber optics using laser excitation.

\section{RESULTS AND DISCUSSION}

\section{Fluorophor selection}

Table 1 lists fluorophors that were tested for susceptibility to quenching by RDX. Of the fluorophors tested, PBA is most susceptible to quenching. This is not unexpected since PBA has a fluorescence lifetime on the order of $150 \mathrm{~ns}$, much longer than typical lifetimes (Chen 1974). This allows more time for quenching to take place. Because of its long lifetime, PBA is also 
highly susceptible to quenching by oxygen and has been used as a reagent for detecting dissolved oxygen (Vaughn and Weber 1970).

Because nitro compounds form charge transfer complexes with amines (Vasundra and Parihar 1980), we hoped that there might be specific interactions that would make amine fluorophors more susceptible to quenching, However, this effect, if it occurs at all, is not large enough to lead to significant quenching.

PBA's fluorescence lifetime is relatively easy to measure because it is long. This means that the extent of quenching is readily related to shortening of the fluorescence lifetime rather than to decreases in intensity. Fluorescence lifetimes are not affected by instrumental drift, slow fluorophor loss because of degradation or leaching and changes in membrane optical properties with time, all of which affect measured intensity values. It will be exceedingly difficult, if not impossible, to calibrate the in-situ optical detection of nitro compounds based on quenching, unless the measured parameter is the fluorescence lifetime.

PBA requires excitation in the ultraviolet, an apparent disadvantage for an application requiring remote excitation through optical fibers. However, PBA absorbs strongly at $337.1 \mathrm{~nm}$, a wavelength at which nitrogen lasers provide short pulses of light with sufficient intensity to overcome transmission losses in optical fibers. We proceeded to use PBA as the fluorophor in subsequent experiments, with the intention of using a nitrogen laser as the source for measuring PBA's fluorescence lifetime remotely through a UV-transmitting optical fiber.

Table 1. Fluorophors tested for quenching by RDX. Data are for ethanol solutions that contain $0.10 \mathrm{mM}$ fluorophor and $200 \mathrm{ppm}$ RDX.

\begin{tabular}{|c|c|c|c|}
\hline \multirow[b]{2}{*}{ Fluorophor } & \multicolumn{2}{|c|}{ Wavelengths ( $\mathrm{nm}$ ) } & \multirow[b]{2}{*}{$\mathrm{I}_{O} / \mathrm{I}$} \\
\hline & Excitation & Emission & \\
\hline Pyrenebutyric acid & 354 & 412 & 1.41 \\
\hline $\begin{array}{l}\text { 5-Dimethylaminonaphthalene-I- } \\
\text { sulfonamidoethyl-trimethyam- } \\
\text { monium iodide }\end{array}$ & 351 & 536 & 1.16 \\
\hline $\begin{array}{l}\mathrm{N} \text {-(5-Dimethylaminonaphthalene-1- } \\
\text { sulfonyl)hexadecylamine }\end{array}$ & 345 & 526 & 1.16 \\
\hline $\begin{array}{l}\text { 11-((5-Dimethylaminonaphthalene-1 } \\
\text { sulfonyl)amino)undecanoic acid }\end{array}$ & d 345 & 526 & 1.16 \\
\hline $\begin{array}{l}\text { 5-Dimethlaminonaphthalene-1-( } \mathrm{N} \text { - } \\
\text { (2-aminoethyl))sulfonamide }\end{array}$ & 346 & 530 & 1.10 \\
\hline $\begin{array}{l}\text { N-Methylacridinium iodide } \\
\text { I-Pyrenemethyldimethylamine }\end{array}$ & 356 & 505 & 1,04 \\
\hline hydrochloride & 337 & 410 & 1.10 \\
\hline
\end{tabular}

\section{Polymer selection}

Table 2 lists polymers that were evaluated as membrane materials, along with comments on the membrane properties. Of the polymers evaluated, only cellulose triacetate and poly(vinyl chloride) yield membranes that stay clear in contact with water and have the right degree of flexibility to be easily handled. However, poly (vinyl chloride) is a poor medium for fluorescence because of the heavy atom effect associated with chloride. Therefore, cellulose triacetate membranes were used for most experiments.

Cyano-bonded stationary phases for high performance liquid chromatography strongly retain nitramines (Jenkins 1989). Therefore, styrene-acrylonitrile copolymer containing $30 \%$ acrylonitrile was evaluated as a membrane material because we hoped that the acrylonitrile would attract more RDX into the membrane, leading to improved sensitivity. Unfortunately, membranes formed from this polymer were not more sensitive than cellulose triacetate membranes and became cloudy upon prolonged exposure to water.

\section{Plasticizer selection}

Plasticizer must be added to produce membranes that are clear and flexible. The plasticizer was also found to have a strong influence on the ability of the membrane to extract RDX from aqueous solution. Table 3 summarizes the results of a preliminary experiment to screen plasticizers for compatibility with RDX by measuring the extent to which $1.0 \mathrm{mg}$ of crystalline RDX dissolved in $0.2 \mathrm{~mL}$ of plasticizer, Of the plasticizers tested, only diethylene glycol dibenzoate and isodecyldiphenyl phosphate (IDP) dissolved all the RDX.

Table 2. Polymers tested as membrane materials. Data obtained using both dioctyl phthalate and isodecyldiphenyl phosphate as plasticizers.

\begin{tabular}{ll}
\multicolumn{1}{c}{ Polymer } & Characteristics \\
\hline $\begin{array}{l}\text { Cellulose Acetate } \\
\text { Cellulose Triacetate }\end{array}$ & Not clear in water. \\
$\begin{array}{l}\text { Polystyrene } \\
\text { Poly(vinyl acetate) }\end{array}$ & Too soft, won't hold shape. \\
$\begin{array}{l}\text { Styrene-acrylonitrile copolymer } \\
\text { (30\% acrylonitrile) }\end{array}$ & $\begin{array}{r}\text { Flexible and clear but tuming } \\
\text { cloudy after two hours in } \\
\text { water. }\end{array}$ \\
\begin{tabular}{l} 
Poly(vinyl chloride) \\
\hline
\end{tabular} & Flexible and clear in water. \\
\hline
\end{tabular}


In a more quantitative experiment, the coefficients for RDX partitioning between dioctyl phthalate and water and between IDP and water were measured to be 7 and 50 respectively. This compares to an octanolwater partition coefficient of 7.65 for RDX (Jenkins 1989). The high partition coefficient for IDP reflects polar interactions between the RDX, a highly polar organic molecule, and the phosphate group on IDP. IDP was therefore used in the majority of membrane formulations evaluated.

We also investigated using 4-cyanophenyl 4heptylbenzoate as a plasticizer. Because cyano-bonded stationary phases for liquid chromatography strongly retain RDX (Jenkins 1989), we hypothesized that a plasticizer with cyano groups might lead to a large RDX partition coefficient. However, although4-cyanophenyl 4-heptylbenzoate is similar in structure to many plasticizers, it did not fully disperse in the polymer and did not produce membranes with improved RDX extraction properties.

\section{Membrane formulation}

The most sensitive membranes were prepared from PBA, IDP and cellulose triacetate. PBA concentration in the membrane is the most important variable affecting response. Figure 4 is a plot of $I_{0} / I$ (unquenched intensity/intensity in presence of quencher) at equilibrium as a function of PBA amount for a membrane prepared using $0.30 \mathrm{~g}$ of cellulose triacetate and 0.10 $\mathrm{mL}$ of IDP. TNT was used as the quencher in this experiment, because the membranes are more sensitive to TNT.

The observation that the extent of quenching increases with the PBA content of the membrane is quite

Table 3. RDX solubility in plasticizers.

\begin{tabular}{ll} 
Plasticizer & Solubility* \\
\hline Diethylene glycol dibenzoate & +++ \\
Glyceryl tri(acetylricinoletate) dibutyl phthalate & ++ \\
Di-(2-ethylhexyl)isophthalate & ++ \\
n-Octyl expoxystearate & ++ \\
Isodecyldiphenyl phosphate & +++ \\
Butylphenyldiphenel phosphate & +++ \\
Ethylhexyldiphenyl phosphate & ++ \\
Di-n-hexylazelate & + \\
n-Propyl oleate & + \\
Dibutyl sebacate & ++ \\
Dioctyl phthalate & ++ \\
Tricresyl phosphate & +++ \\
Tri-butoxyethyl phosphate & ++ \\
\hline & 1.0 mg RDX completely dissolved in $0.2 \mathrm{~mL}$ of plasticizer. \\
*++++ & \\
$+++\quad$ Almost all dissolved. & \\
++ & Partially dissolved.
\end{tabular}

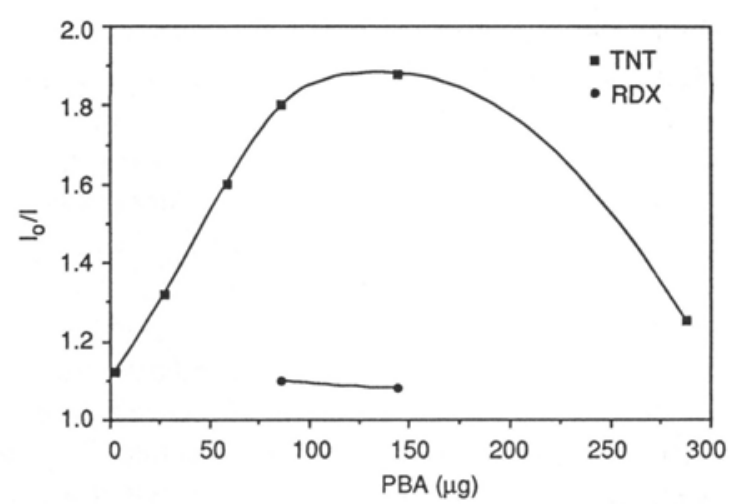

Figure 4. Response of TNT and RDX as a function of the amount of pyrenebutyric acid in a membrane that was prepared using $0.30 \mathrm{~g}$ of cellulose triacetate and $10 \mathrm{~mL}$ of IDP.

surprising. It may mean that the PBA interacts with the nitro compound such that more nitro compound is extracted into the membrane at higher PBA concentrations. The decrease in sensitivity to quenching at the highest PBA concentration tested is not unexpected, since at high PBA concentrations in the membrane, selfquenching may compete with quenching by nitro compounds.

There is no evidence for excimer formation in the emission spectrum for the membrane containing $288 \mu \mathrm{g}$ of PBA in $0.30 \mathrm{~g}$ of cellulose triacetate and $0.10 \mathrm{~mL}$ of IDP. The absence of excimer formation in the membrane is not surprising given the high viscosity of this medium. The fact that polymeric membranes are necessarily highly viscous limits sensitivity because it reduces the probability of an encounter with a quencher while a PBA molecule is excited. Plasticizer content may also affect response, since it should affect both viscosity and partition coefficients.

Table 4 shows raw partitioning data and calculated coefficients for RDX partitioning into selected mem-

Table 4. Partition coefficient data for selected membranes.

\begin{tabular}{|c|c|c|c|c|c|c|c|}
\hline $\begin{array}{c}\text { SAC } \\
(g)\end{array}$ & $\begin{array}{l}\text { CTA } \\
(g)\end{array}$ & $\begin{array}{l}\text { IDP } \\
(m L)\end{array}$ & $\mathrm{A}_{b} \ddagger$ & $\mathrm{A}_{s}$ & $\begin{array}{c}\mathrm{W}_{\text {mem }} \\
(\mathrm{mg})\end{array}$ & $\begin{array}{l}\mathrm{W}_{\text {sol }} \\
\text { (mg) }\end{array}$ & $\mathrm{K}_{p}$ \\
\hline & 0.25 & 0.10 & 0.07 & 0.35 & 3.4 & 3000 & 220 \\
\hline & 0.25 & 0.25 & 0.15 & 0.42 & 4.3 & 3000 & 210 \\
\hline 0.30 & & 0.10 & 0.10 & 0.37 & 3.0 & 3000 & 300 \\
\hline
\end{tabular}

*SAC = styrene-acrylonitrile copolymer; $\mathrm{CTA}=$ cellulose triacetate; IDP = isodecyldiphenyl phosphate.

$\dagger$ See eq 1 for a definition of the column headings. 
brane formulations. The data show that RDX partitions strongly into the membrane. However, given the magnitude of the blank correction and the fact that it was obtained on a separate section of membrane, these data should be regarded as approximate rather than exact.

The partition coefficient is largest for the membrane prepared using the styrene-acrylonitrile copolymer. However, this membrane does not respond as sensitively as membranes prepared from cellulose triacetate.

This suggests that PBA quenches fluorescence more efficiently in plasticized cellulose triacetate. The conditions for partition coefficient measurement are very similar to those under which response to explosives was measured - under the conditions of the measurement, a significant fraction of the explosive is extracted into the membrane, leaving a reduced concentration in the aqueous phase. This means that the sensitivities reported here are somewhat lower than the sensitivity that would be achieved with a ratio of sample volume to membrane volume large enough such that preconcentration in the membrane would not significantly affect the aqueous phase concentration.

\section{Membrane stability}

Two membranes, containing 28 and $144 \mu \mathrm{g}$ of PBA, respectively, showed no measurable loss in fluorescence intensity when continuously irradiated at $337 \mathrm{~nm}$ for 8 hours using the SLM spectrofluorometer.

When ten sections of membrane with a combined weight of $55 \mathrm{mg}$ and PBA content of $20 \mu \mathrm{g}$ were immersed in $2.0 \mathrm{~mL}$ of water, the amounts of PBA leached from the membrane were $2.3,7.4$ and $26 \mathrm{ng}$ after 24,48 and 72 hours respectively.

\section{Rate of response}

Figure 5 shows membrane response to TNT as a function of exposure time. After 40 minutes, response is

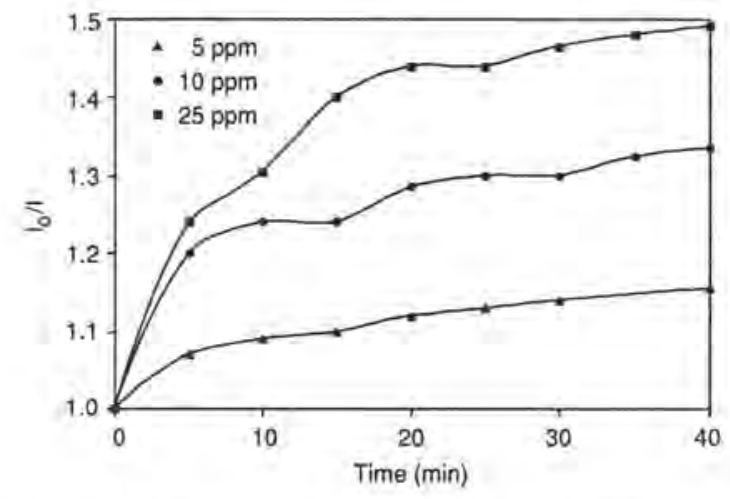

Figure 5. Response vs time for sections of membrane exposed to 5,10 and $25 \mathrm{mg} / \mathrm{L}$ of TNT. approaching a constant value. This is an acceptable rate of response for the intended application.

\section{Reversibility}

Figure 6 shows response vs time for a membrane exposed to $10 \mathrm{ppm}$ of TNT for 30 minutes and then exposed to aqueous blanks. The signal returns towards its initial level when the membrane is exposed again to water.

Coefficients for TNT partitioning into the membranes were not measured. However, TNT has a larger octanol-water partition coefficient than RDX and thus would be expected to partition more strongly into the membrane. The data in Figure 6 show that when the membrane exposed to $10 \mathrm{ppm}$ of TNT is first exposed to water, response decreases to a new steady-state value, which is about $70 \%$ of the maximum signal observed in the presence of TNT. At this point the TNT originally extracted into the membrane has equilibrated between the membrane and the added water. This suggests that the membrane must have extracted approximately $70 \%$ of the TNT from the original aqueous solution under the conditions of the measurement. If the ratio of the volume of the solution to the amount of membrane were increased, then TNT would not be as depleted in the sample, and sensitivity would increase.

\section{Response vs concentration}

Figure 7 shows Stern-Vollmer plots for a membrane prepared using $0.25 \mathrm{~g}$ of cellulose acetate, $0.25 \mathrm{~mL}$ of IDP and $144 \mu \mathrm{g}$ of PBA exposed to varying concentrations of DNT, TNT and RDX. The data for TNT and DNT conform to the Stern-Vollmer law, i.e., the curves are linear with intercepts close to 1.00. With RDX, it's not clear whether the observed data are linear. Detection limits are approximately $2 \mathrm{ppm}$ for TNT and DNT and $10 \mathrm{ppm}$ for RDX.

The major factor affecting relative response to different nitro compounds is the extent to which they partition into the membrane. The sensitivity order correlates with the octanol-water partition coefficients, which are 75.2, 45.0 and 7.65 for DNT, TNT and RDX respectively (Jenkins 1989). There may also be differences in the extent to which various nitro compounds quench fluorescence.

\section{Interferences}

Any substance that partitions into the membrane and quenches fluorescence will interfere with the measurement. Since PBA has previously been used to detect oxygen based on fluorescence quenching (Vaughn and Weber 1970), we expected that dissolved oxygen would interfere. Data confirming this prediction are presented in Table 5 . In the absence of nitro compounds, purging 


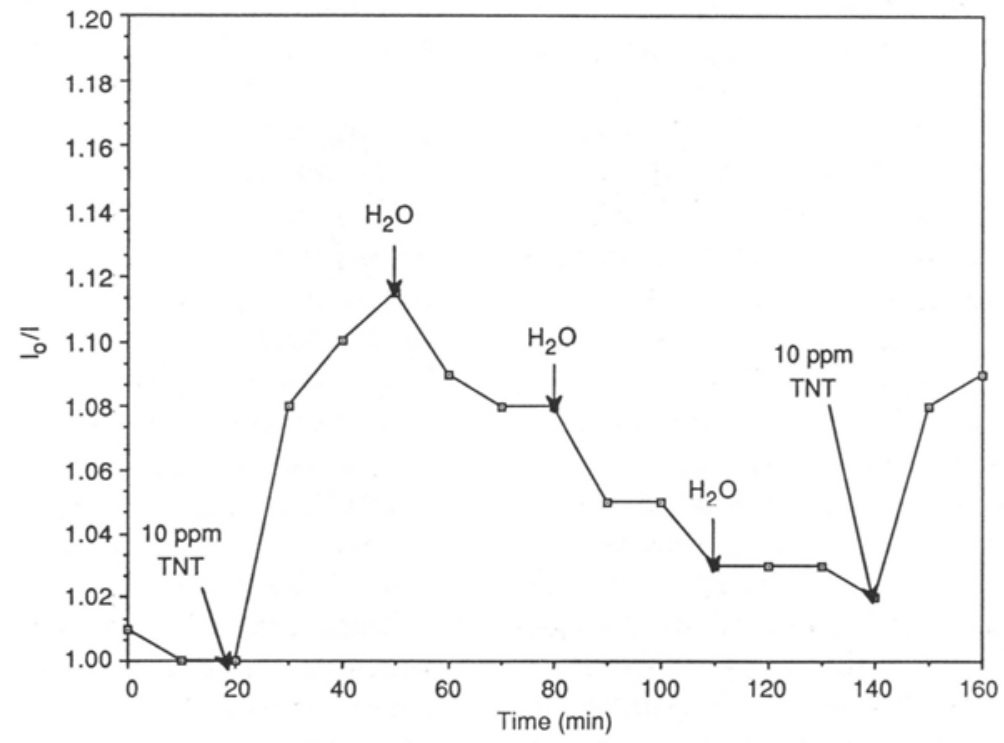

Figure 6. Response vs time for a membrane exposed to $10 \mathrm{mg} / \mathrm{L}$ of TNT, then exposed to water, and then exposed to $10 \mathrm{mg} / \mathrm{L}$ of TNT again.

with nitrogen prior to measuring fluorescence leads to an 8 to $10 \%$ increase in observed intensity. Also, the magnitude of the intensity change for added TNT is greater by a factor of 2 in the absence of oxygen. This should lead to a corresponding improvement in the detection limits quoted above. If this membrane is used for measurements in the field, we strongly recommend that samples be purged with nitrogen prior to the measurement both to enhance sensitivity and to eliminate error owing to variations in dissolved oxygen levels. This can be accomplished in situ by introducing nitrogen through a small-diameter tube at a point near the membrane. (Note that the proposed excitation source for the in-situ measurement is a nitrogen laser, which requires a supply of nitrogen. The same supply of nitrogen could also be used to purge oxygen in the vicinity of the membrane.)

\section{Prospects for improving sensitivity}

The membrane that we have developed is not sufficiently sensitive to detect RDX and TNT levels in contaminant plumes of interest to the U.S. Army. Factors that influence the degree of quenching include the concentration of quencher in the membrane, the rate of the quenching process and the rate of fluorescence (Chen 1974). The faster the rate of the quenching

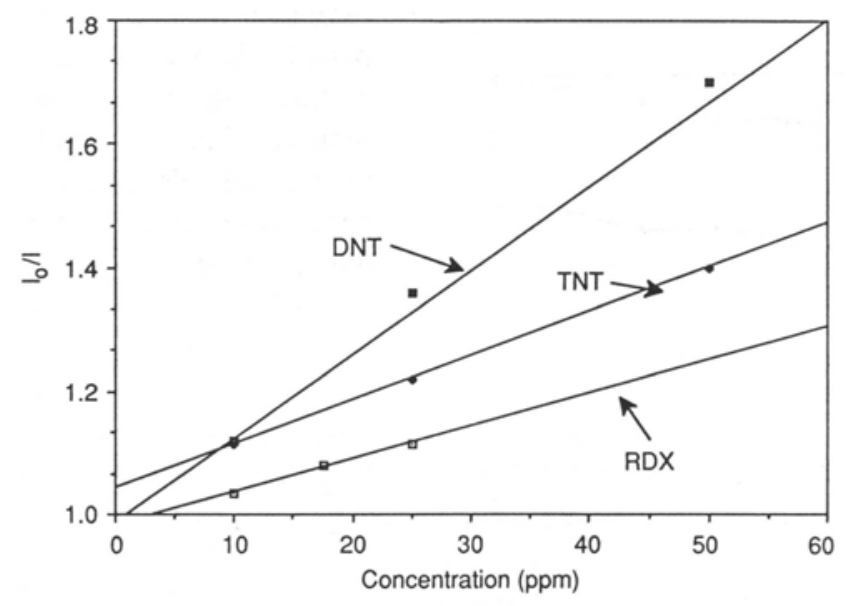

Figure 7. Response vs concentration for RDX, TNT and DNT. 
Table 5. Effect of oxygen on PBA fluorescence (relative intensity).

\begin{tabular}{|c|c|c|c|}
\hline \multicolumn{2}{|c|}{ No TNT } & \multicolumn{2}{|c|}{$10 \mathrm{mg} / \mathrm{L} \mathrm{TNT}$} \\
\hline Air & Nitrogen & Air & Nitrogen \\
\hline 95 & 102 & 87 & 86 \\
\hline
\end{tabular}

process relative to the rate of fluorescence, the more susceptible a fluorophor is to quenching.

For a given aqueous quencher concentration, the equilibrium quencher concentration in the membrane can be increased by developing membranes that more completely extract the quencher. This strategy was successfully pursued in this research. By incorporating polar constituents into the membrane, we developed systems with membrane-water partition coefficients for RDX that are approximately 50 times greater than the octanol-water partition coefficient. Given the success that we have already had with this approach, we doubt that significant further improvements are possible.

The rate of quenching is normally diffusion controlled. In the membrane, however, diffusion is inevitably slow because the medium is necessarily highly viscous. It's not possible to prepare a low viscosity membrane to enhance the rate of quenching,

A given fluorophor has an intrinsic rate of fluorescence. For maximum sensitivity to nitro compounds, the intrinsic rate of fluorescence should be as slow as possible to maximize the time available for nitro compound quenching to compete with fluorescence as a mechanism for electronically excited molecules to return to the ground state. Of the fluorophors tested, PBA was most sensitive to nitro compounds because it has a slow intrinsic rate of fluorescence. PBA fluorescence can also be quenched by components of the medium in which it is located. For example, as shown by the data in Figure 5, oxygen in the membrane quenches PBA fluorescence. Because this competes with quenching by nitro compounds, it reduces sensitivity. Because membrane sensitivity did not directly correlate with the partition coefficient for RDX extraction into the membrane, we believe that PBA fluorescence is quenched by some of the membrane formulations employed in this study. However, we believe that the PBA fluorescence lifetime was close to its intrinsic value in the cellulose triacetate membranes and doubt that significant further improvements insensitivity can be achieved with any other membrane formulation.

The only way that we see to significantly improve sensitivity to RDX is to develop membranes containing a reagent that reacts with $\mathrm{RDX}$ to form a product that retains the quenching properties of RDX and is sufficiently hydrophobic to remain in the membrane. The resulting membranes would be similar to those developed previously for detecting TNT in that the signal would irreversibly increase with time. This would lead to low detection limits by allowing time for sufficient product to accumulate in the membrane. Unfortunately, we have not been able to identify a reaction that meets the necessary criteria.

We, therefore, conclude that it will not be possible to significantly improve detectability beyond the levels achieved in this study,

\section{In-situ calibration}

During the course of this work, considerable thought was given to the problem of in-situ calibration. For reliable measurements it is necessary to be able to distinguish changes in fluorescence intensity caused by quenching from changes caused by instrumental drift, background fluorescence and changes in optical arrangement that may also affect intensity. We originally proposed to address this problem by incorporating into the membrane a reference fluorophor with the following properties. It should be excited at the same wavelength used to excite the fluorophor whose emission is quenched by nitro compounds. It should emit at a different wavelength and should be insensitive to quenching. By measuring quenched intensity relative to emission intensity from the reference fluorophor, we hoped to be able to compensate for drift and other factors affecting absolute intensity values.

We prepared several membranes with two fluorophors. Derivatives of dimethyaminonaphtalenesulfonic acid were used as reference fluorophors, along with PBA, because they are excited at the same wavelength as PBA and emit at longer wavelengths. However, our experiments showed that PBA fluorescence decreased as the concentration of reference fluorophor increased. This means that the reference fluorophor is quenching PBA fluorescence and rendering it less sensitive to quenching by nitro compounds. During the course of the research project, we realized that the problem of in-situ calibration could be better addressed by measuring the fluorescence lifetime. Because quenching provides an alternate pathway for excited states to return to the ground state, the experimentally observed fluorescence lifetime decreases as the extent of quenching increases, as shown in eq 2 (Parker 1968)

$$
t / t_{0}=I / I_{0}
$$

where $t_{0}$ and $t$ are the fluorescence lifetimes in the absence and presence of quencher respectively. Unlike 
intensity, fluorescence lifetimes can be measured with very little drift. Typical fluorescence lifetimes are difficult and expensive to measure because they are on the order of a few nanoseconds. However, PBA's lifetime in water is $115 \mathrm{~ns}$, which is easier to measure (Chen 1974). Furthermore, it can be measured in the presence of shorter-lived background fluorescence,

\section{Proposed system for lifetime measurement}

Nitrogen lasers offer several advantages for the proposed measurement. Because the pulse width is short, they can be conveniently used as a source for measuring the fluorescence lifetime of PBA. The high peak power is important for remote in-situ measurements through fiber optics, because it helps to overcome the problem of light loss because of attenuation in the fiber. Nitrogen lasers are also attractive because they are significantly less expensive than most other types of lasers used as excitation sources in the ultraviolet and visible regions of the electromagnetic spectrum. For example, the Phase-R laser that we used cost only $\$ 2,200$. A problem with nitrogen lasers is that there are significant power variations from pulse to pulse. To address this problem we planned to use a technique developed at Purdue University by F.E. Lytle and colleagues (Desilets et al. 1986, Desilets et al. 1987). The principle of the measurement is illustrated in Figure 8. Two time gated detection channels are used. The time interval between the laser pulse and the opening of the gate differs for the two channels. Under these conditions, the ratio of the intensities measured by the two detection channels varies with fluorescence lifetime. Because both measurements can be made on the same pulse, the intensity ratio is not affected by pulse-topulse fluctuations.

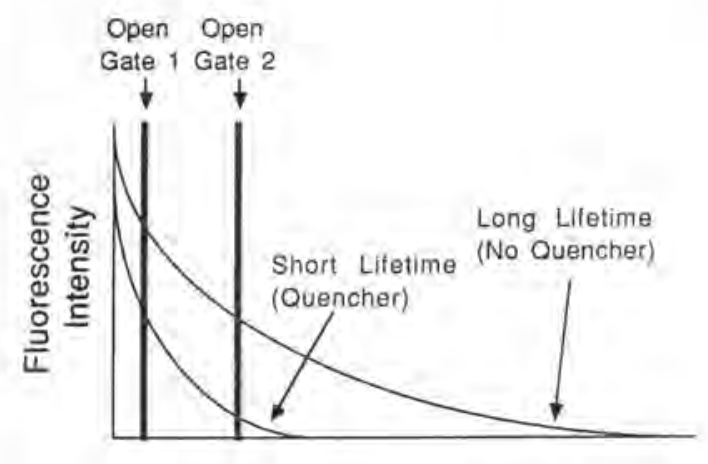

Time

Figure 8. Principle of proposed fluorescence lifetime measurement using a nitrogen laser. The ratio of the signal measured after gate I opens to the signal after gate 2 opens increases as the lifetime gets shorter. Both measurements are made on the same pulse.

\section{Experimental results}

We were able to observe significant fluorescence intensity when exciting membranes through optical fibers. However, we were not able to measure fluorescence lifetimes. The problem is that both the magnitude of response and time constant of the detection electronics depend on the size of the load resistor. The time constant for the detection system is equal to the load resistance times the capacitance of the cable. There is a trade-off between the magnitude of the signal and the time constant of the detection system. As the load resistor gets smaller, the time constant gets shorter but the voltage corresponding to a given photomultiplier current also gets smaller. We were able to observe fluorescence for load resistors as small as $1000 \Omega$. However, using this load resistor, the time constant for the detection system is on the order of $1 \mu \mathrm{s}$. After a pulse from the nitrogen laser, the observed fluorescence decays with a lifetime of approximately $1 \mu \mathrm{s}$, a lifetime that is characteristic of the detection electronics rather than reflecting the fluorescence lifetime of PBA. Therefore, the observed lifetime is not altered by quenching of PBA fluorescence.

We were able to approach conditions necessary for measuring PBA fluorescence lifetimes remotely through fiber optics even though this is not an area where we have previous experience. In the hands of experts, we believe that this measurement is possible with the equipment we used. Even if it is not, the intensity can easily be increased by using a more powerful nitrogen laser, although this would increase the cost of the system. In short, we believe remote in-situ lifetime measurements are feasible even though we failed to demonstrate them ourselves.

\section{CONCLUSIONS}

Nitrated organics are detected by their ability to quench emission from a fluorescent membrane. The sensitivity of a particular compound depends on its tendency to partition into the membrane. This membrane can be used to sense explosives that are present at the parts-per-million level or higher; however, the method is not sufficiently sensitive to detect the concentrations below that level that occur in groundwater. It is unlikely that significant improvements in sensitivity can be realized using the current arrangement.

By measuring changes in fluorescence lifetimes attributable to quenching, it should be possible to measure explosives in water in situ without having to recalibrate the sensor. We were, however, unable to demonstrate this experimentally in this study. 


\section{LITERATURE CITED}

Chen, R.F. (1974) Fluorescence lifetime reference standards for the range 0.189 to 115 nanoseconds. Analytical Biochemistry, 57; 593-604.

Desilets, D.J., J.T, Coburn, D.A. Lantrip, P.T. Kissinger and F.E. Lytle (1986) On-the-fly determination of fluorescence lifetimes from two-point decay measurements. Analytical Chemistry, 58: 1123-1128. Desilets, D.J., P.T. Kissinger and F.E. Lytle (1987) Measurement of fluorescence lifetimes during liquid chromatography. Analytical Chemistry, 59: 1830-1834. Jenkins, T.F. (1989) Development of an analytical method for the determination of extractable nitroaromatics and nitramines in soils. Ph.D. Dissertation, University of New Hampshire (unpublished).

Parker, C.S. (1968) Photoluminescence of Solutions. Amsterdam: Elsevier, p. 71-78.

Vasundra, T.S. and D.B. Parihar (1980) Studies of pyrzaines as their $\mathrm{n}$-pi charge transfer complexes with some nitro aromatic compounds. Journal of Chromatography, 194: 254-261.

Vaughn, W.M. and G. Weber (1970) Oxygen quenching of pyrenebutyric acid fluorescence in water, adynamic probe of the microenvironment, Biochemistry, 9: 464-473.

Zhang, Y. and W.R. Seitz (1989) Single fiber absorption measurements for remote detection of 2,4,6-trinitrotoluene. Analytica Chimica Acta, 221: 1-9

Zhang, Y., W.R. Seitz, D.C. Sundberg and C.L. Grant (1988a) Preliminary development of a fiber optic sensor for TNT. U.S. Army Toxic and Hazardous Materials Agency Report AMXTH-TE-CR-87135.Also CRREL Special Report 88-4.

Zhang, Y., W.R. Seitz and D.C. Sundberg (1988b) Development of a membrane for in-situ optical detection of TNT.U.S. Army Toxic and Hazardous Materials Agency Report AMXTH-TE-CR-88021. Also CRREL Special Report 88-24.

Zhang,Y.,W.R.Seitz,C.L. Grant and D.C.Sundberg (1989a) A clear amine-containing poly (vinyl chloride) membrane for in situ optical detection of 2,4,6tritnitrotoluene. Analytica Chimica Acta. 217:217-227 Zhang, Y., W.R. Seitz and D.C. Sundberg (1989b) Single fiber measurements for remote optical detection of TNT. U.S. Army Toxic and Hazardous Materials Agency Report CETHA-TE-CR-89102. Also CRREL Special Report 89-18. 


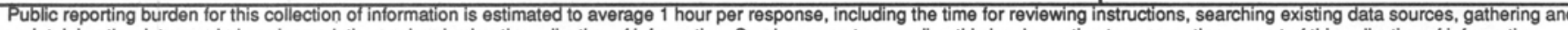
maintaining the data needed, and completing and reviewing the collection of information. Send comments regarding this burden estimate or any other aspect of this collection of information,

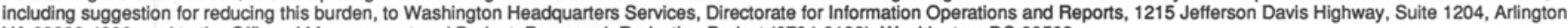
VA 22202-4302, and to the Office of Management and Budget, Paperwork Reduction Project (0704-0188), Washington, DC 20503.

\begin{tabular}{|l|l|l|}
\hline 1. AGENCY USE ONLY (Leave blank) & $\begin{array}{c}\text { 2. REPORT DATE } \\
\text { January 1991 }\end{array}$ & 3. REPORT TYPE AND DATES COVERED
\end{tabular}

\begin{tabular}{|l|l|l|l}
\hline 4. TITLE AND SUBTITLE & 5. FUNDING NUMBERS
\end{tabular}

A Membrane For In-Situ Optical Detection of Organic Nitro Compounds

Based On Fluorescence Quenching

Contract No. DACA89-89-K-0004

6. AUTHORS

W. Rudolf Seitz, Chen Jian and Donald C. Sundberg

7. PERFORMING ORGANIZATION NAME(S) AND ADDRESS(ES)

8. PERFORMING ORGANIZATION REPORT NUMBER

University of New Hampshire

Durham, New Hampshire

9. SPONSORING/MONITORING AGENCY NAME(S) AND ADDRESS(ES)

U.S. Army Cold Regions Research and Engineering Laboratory

72 Lyme Road

Hanover, New Hampshire 03755-1290
U.S. Army Toxic and Hazardous Materials Agency

Aberdeen Proving Ground, Maryland
10. SPONSORING/MONITORING AGENCY REPORT NUMBER

Special Report 91-1

CETHA-TE-CR-91001

11. SUPPLEMENTARY NOTES

12a. DISTRIBUTION/AVAILABILITY STATEMENT

12b. DISTRIBUTION CODE

Approved for public release; distribution is unlimited.

Available from NTIS, Springfield, Virginia 22161.

13. ABSTRACT (Maximum 200 words)

Quenching of emission from fluorescent membranes was evaluated for detecting organo nitro compounds used as explosives. The most sensitive membrane is prepared using solvent casting from cyclohexanone to incorporate pyrenebutyric acid into cellulose triacetate plasticized with isodecyldiphenyl phosphate. The response appears to follow the Stern-Vollmer law for TNT and DNT. The membrane also responds to RDX, but with less sensitivity. Detection limits are approximately 2 ppm for DNT and TNT and $10 \mathrm{ppm}$ for RDX. Attempts were made to adapt the membrane for remote in-situ measurements. In this context, the extent of quenching needs to be determined from the decrease in fluorescence lifetime because this type of measurement is fairly impervious to drift and interference. Fluorescence intensities were measured remotely through fiber optics; however, this was only done when the load resistance in the detection circuit was large, such that the fluorescence decay reflected the RC time constant of the detection electronics rather than the fluorescence lifetime.

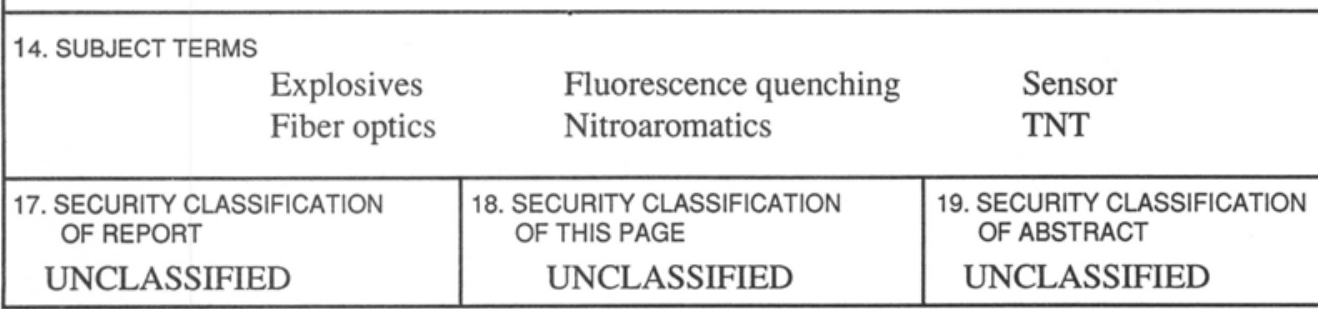

NSN 7540-01-280-5500

\begin{tabular}{|l|}
\hline 15. NUMBER OF PAGES \\
\hline 16 \\
\hline 16. PRICE CODE \\
\hline 20. LIMITATION OF ABSTRACT \\
\hline
\end{tabular}

Standard Form 298 (Rev. 2-89) Prescribed by ANSI Std. Z39-18 